\title{
The effect of recent Venus transit on Earth's atmosphere
}

\author{
Syam S. De $\left({ }^{1}\right)$, B.K. De $\left({ }^{2}\right)$, S.K. Adhikari $\left({ }^{1}\right)$, B.K. Sarkar $\left({ }^{1}\right)$, S.K. Sarkar $\left({ }^{3}\right)$, A. Guha $\left({ }^{1}\right)$, \\ P.K. Mandal $\left({ }^{1}\right)$, S.K. Mandal $\left({ }^{1}\right)$, H.P. Sardar $\left({ }^{1}\right)$ and M. Ray $\left({ }^{1}\right)$ \\ $\left.{ }^{1}\right)$ Centre of Advanced Study in Radio Physics and Electronics, University of Calcutta, Kolkata, India \\ ${ }^{2}{ }^{2}$ Department of Physics, Tripura University, Agartala, India \\ ${ }^{(3)}$ Department of Physics, University of Calcutta, Kolkata, India
}

\begin{abstract}
Some experiments on June 8, 2004, the day of transit of Venus across the Sun, were undertaken at Kolkata (latitude: $22^{\circ} 34^{\prime} \mathrm{N}$ ) to observe the effect, if any, of transit of Venus on FWF, ELF and VLF amplitudes. The result shows a good correlation between their temporal variations during the transit. The observation was unbelievable as the Venus subtends only 1/32th of the cone subtended by Sun on Earth. This anomaly may be explained on the assumption that the height of Venusian atmosphere with high content of $\mathrm{CO}_{2}$, and nitrogen which absorbs electromagnetic and corpuscular radiations from Sun, depleting the solar radiation reaching the Earth to a considerable extent. As a result, relevant parameters of Earth's atmosphere are modulated and here we show how these changes are reflected in identical behaviour of fair weather field and ELF and VLF spectra.
\end{abstract}

Key words Venus transit - effects on atmospheric electric parameters

\section{Introduction}

The quasistatic electric field observed near Earth's surface, i.e. Fair Weather Field (FWF), is maintained by global thunderstorm activities (Bering et al., 1998; Rycroft and Price 2000). Thunderstorm activity and lightning again put the Earth-ionosphere waveguide into resonance producing characteristic spectra in ELF and VLF ranges (Nickolaenko, 1997; Rycroft and Cho, 1998).

Various parameters of the atmospheric electricity are directly related to the global thunderstorm activity and solar radiation.

Mailing address: Prof. Syam S. De, Centre of Advanced Study in Radio Physics and Electronics, University of Calcutta, 1, Girish Vidyaratna Lane, Kolkata 700 009, India; e-mail: de_syam_sundar@yahoo.co.in
The discrete spectra of frequencies $8,14,20 \ldots$ $\mathrm{Hz}$ due to Schumann resonances are generated by electromagnetic emission from the lightning strokes and can be regarded as excitation of an AC global circuit (Bering et al., 1998). Some frequency changes of the peak values and also amplitude changes are found to be present in the observed data which are attributed by the uncertainties arising from spatial distribution of lightning sources exciting the Schumann resonance modes (De et al., 2004). Schumann resonance phenomenon as well as their measurement techniques and other aspects have been described by many workers (Tzanis and Beamish, 1987; Williams, 1992; Satori, 1996; Huang et al., 1999).

In three areas, namely, atmospheric potential gradient, ELF and VLF, we are taking continuous records where some significant deviations are observed in the values of the involved parameters during the transit of Venus on June 8,2004 . The transit started at 10:44 h local time and continued up to $16: 53 \mathrm{~h}$ local time, which was visible from Kolkata (latitude $22^{\circ} 34^{\prime} \mathrm{N}$ ) throughout this period. The local sunrise and 
sunset times were 04:52 $\mathrm{h}$ and 18:22 $\mathrm{h}$ respectively. This paper presents the results of experimental observations during Venus transit.

\section{Experimental arrangement}

We take observations from the roof top at a height of about $26 \mathrm{~m}$ from ground. The vertical electric field is measured with an ac field-mill which has an aluminum rotor plate of $12 \mathrm{~cm}$ in diameter. The output from the amplifier is recorded through computer sound card at a sample rate of 44100 per second. The rms value of the recorded signal is used to find the required electric field from the calibration chart. We calibrated the field-mill in a vertical field setup between two large aluminum cover plates, electrically isolated at a given potential through a fixed distance between them. The outer shield of the field-mill is grounded properly to ensure possible field distortions. The sensitivity of the fieldmill is $(0.33 \pm 0.03) \mathrm{Vm}^{-1}$.

For the observation of Integrated Field Intensity (IFI) of VLF Sferics at $9 \mathrm{kHz}$, an 8 SWG straight copper wire of $120 \mathrm{~m}$ in length is used as the antenna. The antenna is sensitive to the vertical electric field component of the electromagnetic signal. It is installed horizontally at a height of about $30 \mathrm{~m}$ above the ground. The signal processor is tuned to the desired frequency. The overall $Q$-factor of the tuning circuit is around 300 . The signal from the tuning stage is rectified and fed to a log amplifier. The time constant of the output from the signal processor is $10 \mathrm{~s}$. The data are recorded using a $12 \mathrm{bit}$ $\mathrm{ADC}$ at a sample rate of 5 .

For observations on Schumann resonances, two square loop antennas in series combination have been used. The length of each side of one of the loops is $1 \mathrm{~m}$ with a total of 75 turns and other with 90 turns having a length of $1.3 \mathrm{~m}$ for each side. The antennas are sensitive to the magnetic field component of the natural Schumann resonance electromagnetic signals. A stereopreamplifier-integrated circuit with LA3161 chip, whose low frequency response starts below $5 \mathrm{~Hz}$, has been used to pre-amplify the signal from antenna. The chip is capable of detecting signals in the microvolt region. The pre-am- plified signal is then amplified with a low pass amplifier with upper cut-off at $35 \mathrm{~Hz}$. The amplified signal is recorded using computer sound card at a sample rate of 44100 per second.

\section{Analyses of the observed data}

\subsection{Schumann resonances}

The temporal variations of the amplitudes of first three Schumann resonance modes on 8th June 2004, during the Venus transit, together with their averaged values of 18 days are shown in fig. 1. Standard deviations from the average values have been plotted as error bars in the average plot. Standard deviations have been calculated over 2nd and 3rd quartile of the frequency distribution curve. It is seen that the average amplitude of the first mode line in the average value graph is $0.025 \mathrm{AU}$ (Arbitrary Unit) except almost a sharp 3 fold increase at $14 \mathrm{~h}$ local time, whereas on the day of transit, the corresponding amplitude decreases gradually from $10 \mathrm{~h}$ to $16 \mathrm{~h}$ from $0.06 \mathrm{AU}$ to $0.03 \mathrm{AU}$. After $16 \mathrm{~h}$, the amplitude increases. The second mode amplitude in the average value graph remains steady at 0.01 AU except almost a two fold increase at $14 \mathrm{~h}$ local time. On the day of transit, the corresponding amplitude shows the gradual decrease from $0.035 \mathrm{AU}$ to almost zero at $13 \mathrm{~h}$ local time which then gradually increases. The third mode amplitude in the average graph remains the same except a depression between $13 \mathrm{~h}$ to $14 \mathrm{~h}$ local time, whereas on the day of transit, the amplitude decreases from $10 \mathrm{~h}$ local time to $13 \mathrm{~h}$ local time and shows a peak at $14 \mathrm{~h}$ and then the amplitude decreases up to $15 \mathrm{~h}$ local time and then again increases.

\subsection{VLF atmospherics}

A comparative picture of the IFI at $9 \mathrm{kHz}$ atmospherics on 8th June 2004, during the Venus transit (continuous line) and its 18 days' average value over the same period (dotted line) is presented in fig. 2. The dotted grey lines represent standard deviation. On the day of transit, there are some unique peaks that appear just before 


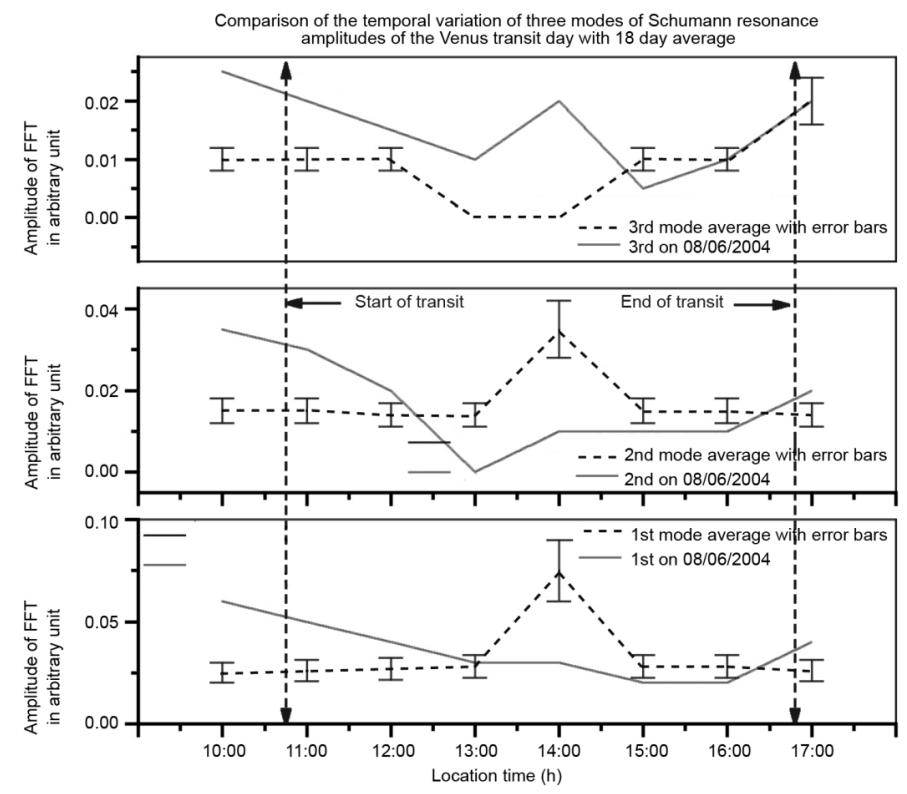

Fig. 1. Variation of peak magnetic amplitude of the three modes of Schumann resonance spectra on the day of Venus transit, 8th June 2004 (continuous line) and average variation of the same parameter around 8th June for 18 days (dotted line).

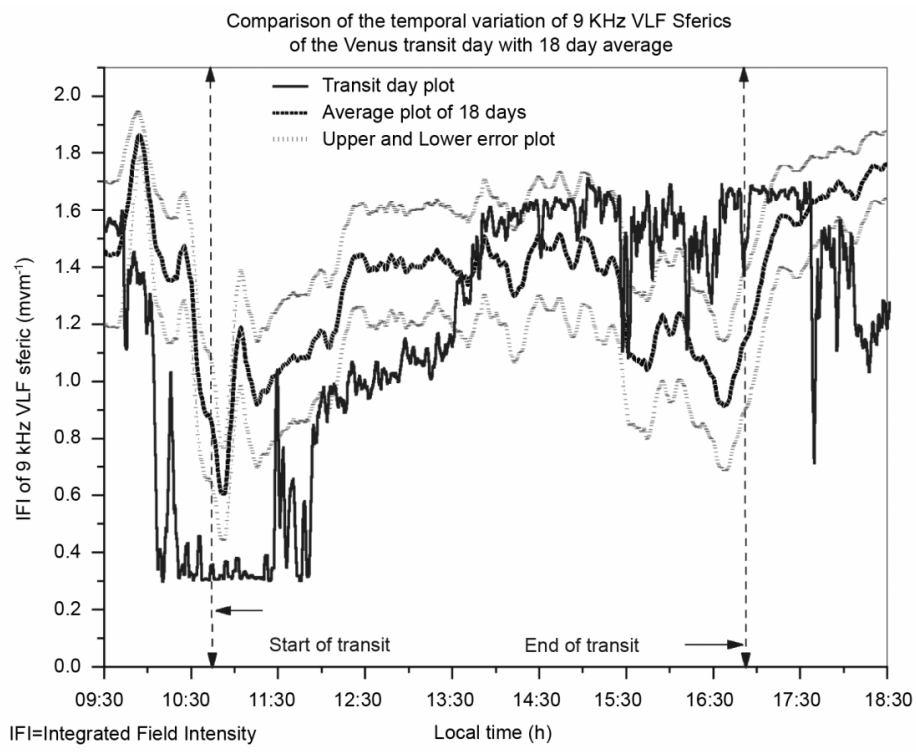

Fig. 2. Variation of atmospheric Integrated Field Intensity (IFI) at $9 \mathrm{kHz}$ VLF on the day of Venus transit, 8th June 2004 (continuous line) and average variation of the same parameter around 8th June for 18 days (dotted line) and the dotted grey lines represent standard deviation. 


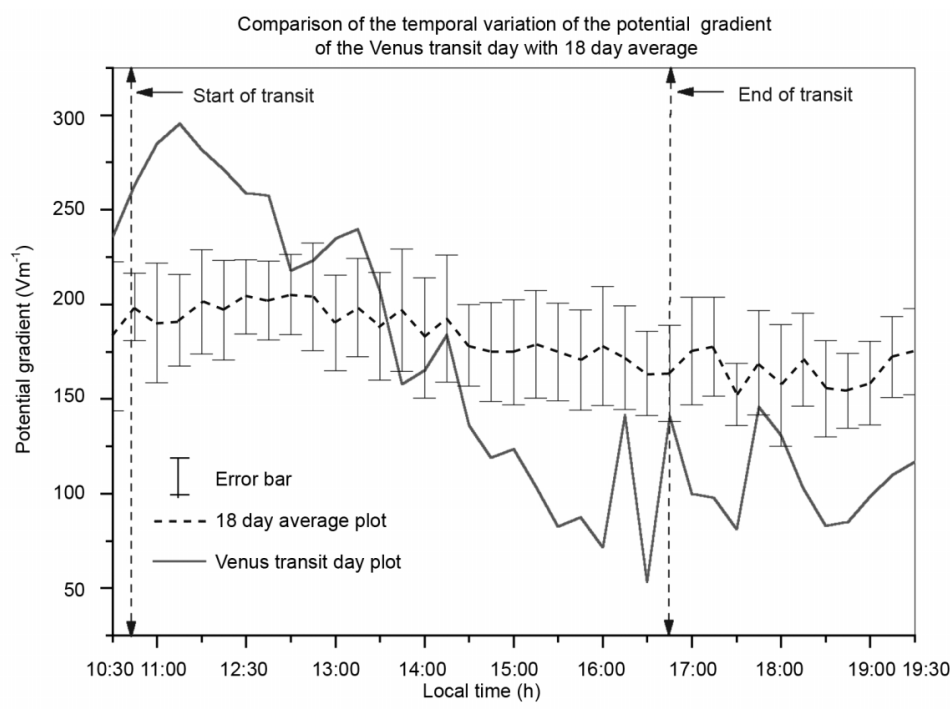

Fig. 3. Variation of atmospheric vertical potential gradient on the day of Venus transit, 8th June 2004 (continuous line) and average variation of the same parameter around 8th June for 18 days (dotted line).

and after the beginning of the transit. The peaks are in decreasing order of magnitude. The IFI then slowly increases to a steady value showing considerable fluctuations before and after the end of the transit also.

It is worth mentioning that in both the measurements of potential gradient and VLF IFI, just after the beginning of transit and before the end of transit, their values lie beyond the standard deviation limits of the average variation. In case of potential gradient, it decreases from higher value to a lower value whereas in case of VLF IFI, it increases from lower value to a higher value. In Schumann resonance also, we obtained a similar trend.

\subsection{Potential gradient}

Figure 3 represents the temporal variation of vertical potential gradient on 8th June, 2004 during the Venus transit (continuous line) and its values averaged over other 18 days around that date (dotted line) with standard deviations from the average value plotted as error bars. Firstly, we notice that the maximum and minimum values of the potential gradient on the day of transit are around $300 \mathrm{Vm}^{-1}$ and $50 \mathrm{Vm}^{-1}$ respectively. But during the same period on other days, it is around $205 \mathrm{Vm}^{-1}$ and $152 \mathrm{Vm}^{-1}$, respectively. The high values owe to the higher level of pollution at the place. Secondly, the curve during the transit shows significant deviation from the average values since the variation is well beyond the standard deviation of the average trend. There is sudden enhancement of potential gradient up to around $300 \mathrm{Vm}^{-1}$ after the start of the transit and then the gradual decrease extends up to around $50 \mathrm{Vm}^{-1}$.

In the cases of potential gradient and VLF Sferics and also in the case of first and second mode of Schumann resonance, the «effect» of the Venus transit reverses its nature at about $1400 \mathrm{~h}$ local time (figs. 1-3). This is probably due to the contribution from regional thunderstorm centre in SE Asia. The behaviour of the third mode of Schumann resonance curves are not properly understood (fig. 1).

The Atmospheric temperature recorded at an Indian Meteorological Department observatory about $10 \mathrm{~km}$ from observation site did not show any outstanding change during transit. Relative humidity was a little bit higher than normal throughout the day. 


\section{Discussion}

The commonly accepted model explains FWF near the ground by the dynamic equilibrium between charging currents from lightning activity and the leakage current of the Earthionosphere spherical capacitor (Bering et al., 1998; Fullekrug et al., 1999; Rycroft and Price, 2000). The idea is corroborated by the measurements of Fullekrug et al. (1999) who showed a similarity in the monthly averaged pattern of FWF and ELF amplitude.

During the transit of Venus, it obstructs 1/1000th part of incident solar radiation coming to the Earth, since the angular diameter of Venus compared to that of the Sun as seen from the Earth is of the ratio 1:32. Therefore, apparently the transit of Venus should have no significant effect on any terrestrial phenomenon. But, in spite of this fact, the changes observed in the atmospheric parameters at Kolkata during the transit of Venus are quite significant. This anomaly may be explained in terms of very large extent of the atmosphere of Venus with about $96 \% \quad \mathrm{CO}_{2}$ that produces atmospheric pressure of about 92 Bars (http://pr.erau.edu/ -holmesg). Because of very high content of $\mathrm{CO}_{2}$, and nitrogen (Warby, 1943), its atmosphere absorbs high frequency radiations, protons as well as other ionizing particles from the Sun thereby depleting to a considerable extent the solar radiation reaching the Earth. Hence, during the transit, there will be some changes in the depth of interaction between Earth's magnetosphere and solar radiations. It is expected that collision frequency would be affected little by such interaction. The main influence would be on the rate of ionization within the Earth-ionosphere cavity. This in turn introduces perturbation in the atmospheric electrical conductivity (Roble, 1991) which may give rise to the changes that have been observed. The modification of solar atmosphere under the influence of tidal forces from the Venus during transit may also contribute the observed effects (Harnischmacher and Rawer, 1981).

Each plot has two distinct time zones, one up to $13: 30$ to $14: 00 \mathrm{~h} \mathrm{LT}$ and other after that. A prominent feature of our observation is that the deviation of the average values of measured parameters changes signs at the boundary of two time zones.

Atmospheric potential gradient begins to increase from $2 \mathrm{~h}$ before onset, reaches a maximum after $1 \mathrm{~h}$ from onset and then gradually decreases and reaches a minimum at almost the same time as the end of transit. Then it gradually increases and becomes equal to the average value about $4 \mathrm{~h}$ after the end of transit. The beginning and end of transit mean the transit of solid core of Venus. The times of 1 st and 4th contact of atmosphere of Venus are different than the times of transit, 1st contact being earlier and 4th contact later than the recorded time of transit. The cause of the observed phenomenon may probably due to the rise of lower boundary of the ionosphere. Changes of convection current due to change in intensity of solar radiation may also contribute to the cause. Of particular interest is the increase in potential gradient from sunrise which continues upto $1 \mathrm{~h}$ after onset of transit. A similar observation in case of solar eclipse is known (Dhanorkar et al., 1989).

The amplitudes of 3 modes of Schumann resonance show gradual increase from average value from sunrise and this tendency decreases from $10 \mathrm{~h} \mathrm{LT}$, i.e. one hour before transit. The gradual decrease continues up to $13 \mathrm{~h} \mathrm{LT}$, after which the variations assume the characteristics of regional thunderstorm activity in Asia (Nickolaenko and Hayakawa, 2002).

Amplitudes of $9 \mathrm{kHz}$ Sferics descend from the average value about $1 \mathrm{~h}$ before transit and then it slowly rises after $1 \mathrm{~h}$. It crosses the average graph gradually and again meets it after 1 hour from the end of transit (fig. 2). At the end, it shows a fluctuation which may have its origin in local thunderstorm activity. The amplitude is significantly lower from about $1 \mathrm{~h}$ before transit to about $14 \mathrm{~h} \mathrm{LT}$. This portion of the IFI curve is structurally similar to the average curve. Apparently, Venus transit affects the attenuation parameter. For its explanation, calculation of amplitude under «eclipse» condition is necessary.

Atmospheric potential gradient on the day of transit of Venus just before its onset increases which is probably due to the scattering of solar radiation and ionizing solar particles by the protruding part of Venusian atmosphere (fig. 3). After the onset of transit, absorption of solar ra- 
diation takes over causing gradual decrease of FWF, FFT amplitude and IFI [fig. 1 (continuous line), fig. 2 (continuous line) and fig. 3 (continuous line)]. The peak around $1400 \mathrm{~h}$ local time in fig. 1 correlates with lightning activity of regional thunderstorm centre in Asia (Nickolaenko and Hayakawa, 2002). It is interesting to note that all the modes of Schumann resonance do not respond equally to the lightning activity, probably because of differences in non linear interaction with transverse resonance and differences in attenuation rates.

\section{Conclusions}

The transit of Venus is an event encouraging detailed study of its terrestrial effects. Observations on Schumann resonance spectra and Earth's atmospheric electric field were undertaken during the transit of Venus. The results have been compared with our ongoing records. How so small a speck in the backdrop of solar disc can cause so large effect is an open question. We have tried to explain this anomaly in this presentation.

\section{Acknowledgements}

The work has been funded by Indian Space Research Organization (ISRO) through S. K. Mitra Centre for Research in Space Environment, Institute of Radio Physics and Electronics, University of Calcutta, Kolkata 700 009, India.

\section{REFERENCES}

Bering, E.A. III, A.A. Few and J.R. Benbrook (1998): The global electric circuit, Physics Today, 51, 24-30.
DE, S.S., A.K. SAHA and M. DE (2004): Measurement of ELF emission in the upper atmosphere over Kolkata due to Schumann resonances, Ind. J. Radio Sp. Phys., 33, 32-34.

Dhanorkar, S., C.G. DeshPande and A.K. Kamra (1989): Atmospheric electricity measurements at Pune during solar eclipse of 18th March 1988, J. Atmos. Terr. Phys., 91, 1031-1034.

Fullekrug, M., A.C. Fraser Smith, E.A. Bering and A.A. FEW (1999): On the hourly contribution of global cloud to ground lightning activity to the atmospheric electric field in the Antarctic during December 1992, J. Atmos. Solar Terr. Phys., 61, 745-750.

HARNISCHMACHER, E. and K. RAWER (1981): Lunar and planetary influences upon the peak electron density of the ionosphere, J. Atmos. Terr. Phys., 43, 643-648.

Huang, E., E. Williams, R. Boldi, S. Heckman, W. Lyons, M. TAYlor, T. Nelson and C. WONG (1999): Criteria for sprites and elves based on Schumann resonance observations, J. Geophys. Res., 104, 16943-16964.

NickolaEnKo, A.P. (1997): Modern aspects of Schumann resonance studies, J. Atmos. Solar-Terr. Phys., 59, 805816.

Nickolaenko, A.P. and M. Hayakawa (2002): Resonances in the Earth Ionosphere Cavity (Kluwer Academic Publisher), p. 97.

RoBLE, R.G. (1991): On modeling component processes in the Earth's global electric circuit, J. Atmos. Solar-Terr. Phys., 53, 831-847.

RYCROFT, M.J. and M. CHO (1998): Modelling electric and magnetic fields due to thunderclouds and lightning from cloud top to the ionosphere, J. Atmos. Solar Terr. Phys., 60, 889-893.

RyCROFT, M.J. and C. PRICE (2000): The global atmospheric electric circuit, solar activity and climatic change, $J$. Atmos. Solar-Terr. Phys., 62, 1563-1576.

SATORI, G. (1996): Monitoring Schumann resonances, II. Daily and seasonal frequency variations, J. Atmos. Terr. Phys., 58, 1483-1488.

Tzanis, A. and D. BEAmish (1987): Time domain polarization analysis of Schumann resonance waveforms, J. Atmos. Terr. Phys., 49, 217-228.

WARBY, R.E. (1943): Absorption spectrum of $\mathrm{N}_{2}$ in the extreme ultraviolet, Phys. Rev., 64 (7-8), 207-224.

Williams, E.R. (1992): The Schumann resonance: a global tropical thermometer, Science, 256, 1184-1187.

(received November 29, 2005; accepted July 28, 2006) 